\title{
EFEITO DE FUNGICIDAS TRIAZÓIS SOBRE O CONTROLE DA CERCOSPORIOSE EM MUDAS DE CAFEEIRO
}

\author{
F.R.A. Patricio ${ }^{1}$; M.T. Braghini ${ }^{2}$ \\ ${ }^{1}$ Instituto Biológico, Centro Experimental Central, CP 70, CEP 13012-970, Campinas, SP, Brasil. E-mail: \\ flavia@biologico.sp.gov.br
}

\section{RESUMO}

Fungicidas do grupo dos triazóis e suas misturas com inseticidas são convencionalmente empregados para o controle da ferrugem e do bicho mineiro na cultura do cafeeiro. Neste estudo avaliou-se o efeito desses produtos sobre o controle da cercosporiose, causada por Cercospora coffeicola, outra importante doença do cafeeiro. Três experimentos foram realizados em casa de vegetação com mudas de cafeeiro da cultivar Mundo Novo IAC 501, sendo os produtos aplicados na parte aérea e no substrato. Os produtos aplicados na parte aérea foram: epoxiconazol, epoxiconazol + piraclostrobina, tetraconazol, ciproconazol, oxicloreto de cobre, azoxistrobina e carbendazim e no substrato: ciproconazol + tiametoxam, triadimenol + dissulfotom e triadimenol. Testemunhas sem tratamento químico também foram incluídas nos três experimentos. As mudas foram inoculadas com suspensões preparadas com cinco isolados de C. coffeicola (3-4 conídios/ $\mathrm{mL}$ ). A incidência e a severidade da doença foram avaliadas de 20 até 56 dias após as inoculações. Com exceção do oxicloreto de cobre, todos os demais tratamentos foram eficientes no controle da cercosporiose. Os triazóis, com exceção do tetraconazol, apresentaram sintomas reversíveis de fitotoxicidade. Os produtos: ciproconazol + tiametoxam, epoxiconazol + piraclostrobina e carbendazim tiveram os maiores efeitos residuais, e mantiveram o controle da doença por até 56 dias após sua aplicação. Os triazóis utilizados para o controle da ferrugem também são eficientes para o controle da cercosporiose do cafeeiro.

PALAVRAS-CHAVE: Cercospora coffeicola, controle químico, Coffea arabica, Hemileia vastatrix, Leucoptera coffeicola.

\begin{abstract}
EFFECTIVENESS OF TRIAZOLE FUNGICIDES FOR THE CONTROL OF BROWN EYE SPOT ON COFFEE SEEDLINGS. Triazole fungicides and their mixtures with insecticides are usually employed for the control of coffee rust and leaf miner in coffee crops. In this study these chemicals were evaluated for the control of brown eye spot, caused by Cercospora coffeicola, another important disease of this crop. Three experiments were carried out in a greenhouse with coffee seedlings of the cultivar Mundo Novo IAC 501. The fungicides epoxiconazole, epoxiconazole + pyraclostrobin, tetraconazole, ciproconazole, copper oxychloride, azoxystrobin and carbendazim were applied to the leaves of coffee seedlings, and ciproconazole + tiametoxam, triadimenol + dissulfotom, and triadimenol were applied to the seedlings substrate. Controls were also included in all experiments. The seedlings were inoculated with suspensions prepared with a mixture of five C. coffeicola isolates (3-4 conidia/mL). The incidence and severity of the disease were evaluated 20 to 56 days after the inoculations. With the exception of copper oxychloride, all products were efficient for the control of brown eye spot. The triazoles, with the exception of tetraconazole, showed reversible phytotoxic effects. Ciproconazole + tiametoxam, epoxiconazole + pyraclostrobin and carbendazim had the highest residual effects and maintained small levels of the disease until 56 days after their application. The triazoles used for the control of coffee rust are also effective for the control of brown eye spot.

KEY WORDS: Cercospora coffeicola, chemical control, Coffea Arabica, Hemileia vastatrix, Leucoptera coffeicola.
\end{abstract}

2Instituto Agronômico, Centro de Análise e Pesquisa Tecnológica do Agronegócio do Café “Alcides Carvalho", Campinas, SP, Brasil. 


\section{INTRODUÇÃO}

A cercosporiose, também conhecida como mancha de olho pardo, é causada por Cercospora coffeicola Berk. \& Cooke e está presente em todas as regiões produtoras de café do Brasil (Godoy et al., 1997). A cercosporiose pode ser considerada uma das principais doenças da cultura em várias regiões produtoras, como no cerrado (CARVALHO et al., 2002), e nas demais regiões cafeicultoras a sua importância tem aumentado (THOMAZIELLO et al., 2000). A doença causa lesões nas folhas, que caem rapidamente (Godor et al., 1997), pode derrubar os frutos em expansão (MATIELLO, 1991) e prejudicar a qualidade da bebida (GoDor et al., 1997; ZAMBOLIM et al., 1997). Em viveiros, a desfolha intensa pode atrasar o desenvolvimento das mudas (ZAMBOLIM et al.,1997). Cafezais conduzidos em solos arenosos, ou com nutrição deficiente ou desequilibrada, especialmente na relação N/K, e lavouras expostas ao sol da tarde, podem ser mais atacados pela cercosporiose (Zambolim et al., 1997; CARvalho et al., 2002; PozZA et al., 2000; ZамвоLIM et al. 2005).

As medidas culturais são muito importantes para o controle da cercosporiose e têm início pela produção de mudas sadias, sob substrato adubado adequadamente, em viveiro bem instalado. Nos viveiros, o controle químico é muitas vezes necessário, especialmente antes da aclimatação das mudas para serem levadas ao campo (ZамвоLim et al., 1997; ZАмвоLiм et al., 2005). Em condições de campo recomenda-se evitar solos arenosos e manter rigoroso controle das adubações durante todo o ciclo da cultura (ZАMBolim et al., 1997; ZАмвоLIM et al., 2005), entretanto, a utilização de fungicidas para o controle da cercosporiose é parte integrante dos programas de tratamento fitossanitário do cafeeiro (THOMAZIELlo et al., 2000). Para o controle químico da cercosporiose podem ser utilizados fungicidas cúpricos, benzimidazóis e estrobilurinas (Pozza et al., 1997; ZамвоLim et al., 2005; PAtricio et al., 2008).

Embora a cercosporiose esteja presente no campo durante o ano todo, ela causa os maiores prejuízos durante o período de formação do fruto, que vai de janeiro a abril/maio. Coincidentemente, nesse período, tem início a expansão do ciclo da ferrugem, causada por Hemileia vastatrix Berk. Et Br., considerada a mais importante doença do cafeeiro no Brasil. Como grande parte das lavouras do parque cafeeiro brasileiro, mais de $90 \%$, é formada com cultivares suscetíveis à ferrugem (MATIELlo; Almeida, 2006), o controle químico dessa doença é rotineiramente realizado na cultura do café, e a maioria dos programas de tratamento com fungicidas propõe o controle simultâneo da ferrugem e da cercosporiose.
As recomendações para o controle químico da ferrugem tiveram início com os fungicidas cúpricos, na década de 1970, e passaram a se basear no uso de triazóis via solo, principalmente nas décadas de 1980 a 1990. Na década seguinte, foram desenvolvidas as tecnologias que empregavam triazóis via foliar. Atualmente, ocontrolequímico da ferrugem se baseia no uso de misturas de triazóis com estrobilurinas (Matiello; Almeida, 2006). Este último grupo de fungicidas foi incluído no manejo da cultura para aumentar a eficiência do controle químico da ferrugem e incluir o controle da cercosporiose (MATIELLO; AlmeidA, 2006).

Um grande número de trabalhos foi conduzido visando avaliar o controle da ferrugem, bem como o controle simultâneo da ferrugem e da cercosporiose em condições de campo com triazóis aplicados via foliar e no solo. Entretanto, em condições de campo é difícil estimar o efeito exclusivo dos fungicidas do grupo dos triazóis sobre o controle da cercosporiose do cafeeiro, especialmente porque muitos fatores interferem nos resultados, como as elevadas incidências de ferrugem, que podem dificultar a detecção da cercosporiose nas folhas, as condições nutricionais das plantas, o manejo da lavoura, entre outros. Este trabalho foi realizado com o objetivo de avaliar, em condições controladas, o efeito de fungicidas do grupo dos triazóis, além de um cúprico, um benzimidazol e uma estrobilurina, sobre o controle da cercosporiose do cafeeiro.

\section{MATERIAL E MÉTODOS}

No presente estudo foram conduzidos três experimentos com mudas de cafeeiro, do cultivar Mundo Novo IAC 501, em condições de casa de vegetação e em viveiro, no Instituto Agronômico em Campinas, SP. Todas as mudas utilizadas foram produzidas segundo as recomendações técnicas (THOMAZIELLO et al., 2000).

No primeiro ensaio, as mudas, com oito meses de idade, receberam os tratamentos com os seguintes fungicidas e suas respectivas doses de produto comercial e do princípio ativo por $100 \mathrm{~L}$ de água: epoxiconazol (120 mL p.c.e $15 \mathrm{~g}$ p. a.), epoxiconazol + piraclostrobina $(300 \mathrm{~mL}$ de p.c. e $15+39,9 \mathrm{~g}$ de p.a., respectivamente), tetraconazol $(200 \mathrm{~mL}$ de p.c. e $20 \mathrm{~g}$ de p.a.), ciproconazol (100 $\mathrm{mL}$ de p.c. e $10 \mathrm{~g}$ de p.a.), oxicloreto de cobre (840 g de p.c. e $420 \mathrm{~g}$ de p.a.), azoxistrobina ( $20 \mathrm{~g}$ de p.c. e $10 \mathrm{~g}$. de p.a.) e carbendazim ( $200 \mathrm{~mL}$ de p.c. e $100 \mathrm{~g}$ de p.a.), além da testemunha, com aplicação apenas de água. Em todos os experimentos os produtos foram aplicados na parte aérea por meio de pulverizador costal manual, sob pressão de $\mathrm{CO}_{2}$, com bico tipo leque, dois a três dias antes da inoculação do patógeno. 
Osegundo experimento foi realizado com mudas de um ano de idade, mantidas em recipientes contendo três de litros de substrato, preparado de acordo com as recomendações técnicas (THOMAZIELLo et al., 2000). Nesse ensaio foram realizados tratamentos via solo com os seguintes fungicidas e suas respectivas doses de produto comercial e do princípio ativo por muda: ciproconazol + tiametoxam $(2,0 \mathrm{~g}$ de p.c. e $0,6+0,6$ g de p.a), triadimenol $(0,8 \mathrm{~g} /$ planta e 0,005 g. de p.a.), ou na parte aérea, com os fungicidas e suas respectivas doses de produto comercial e do princípio ativo por $100 \mathrm{~L}$ de água: azoxistrobina (20 g de p.c., 10 g. de p.a./ $100 \mathrm{~L}$ de água) e oxicloreto de cobre (840 g de p.c., $420 \mathrm{~g}$ de p.a./100 L de água). A testemunha recebeu apenas água. Os produtos de solo foram aplicados 15 dias antes da inoculação, sendo metade da dose enterrada de cada lado da planta, a $5 \mathrm{~cm}$ de profundidade.

No terceiro ensaio, mudas com sete meses de idade, formadas em recipiente plástico contendo 1,5 L de substrato, receberam, via substrato, os seguintes tratamentos e suas respectivas doses de produto comercial e do princípio ativo por muda: triadimenol + dissulfoton (1,5 g de p.c. ou 0,00225 g + 0,01125 g. de p.a., respectivamente/planta), ciproconazol + tiametoxam $(1,0 \mathrm{~g}$ de p.c./planta ou $0,3+0,3 \mathrm{~g}$ de p.a), triadimenol ( 0,4 g ou 0,0025 g de p.a./planta). Os tratamentos na parte aérea e suas respectivas doses de produto comercial e do princípio ativo por $100 \mathrm{~L}$ de água foram: epoxiconazol (120 mL p.c. e $15 \mathrm{~g}$ p. a.), epoxiconazol + piraclostrobina $(300 \mathrm{~mL}$ de p.c. e $15+39,9$ gde p.a., respectivamente), tetraconazol (200 mL de p.c. e $20 \mathrm{~g}$ de p.a.), ciproconazol (100 $\mathrm{mL}$ de p.c. e $10 \mathrm{~g}$ de p.a.), oxicloreto de cobre (840 $\mathrm{g}$ de p.c. e $420 \mathrm{~g}$ de p.a.), azoxistrobina (20 g de p.c. e 10 g. de p.a.) e carbendazim ( $200 \mathrm{~mL}$ de p.c. e 100 g de p.a.), além da testemunha com aplicação de apenas água. Os produtos foram aplicados no solo 14 dias antes da primeira inoculação, sendo metade da dose enterrada de cada lado da planta, a $5 \mathrm{~cm}$ de profundidade, e na parte aérea, três dias antes da primeira inoculação.

Nos três ensaios as mudas foram inoculadas com uma suspensão composta pela mistura, em partes iguais, de cinco isolados de C. coffeicola, obtidos em diferentes regiões do Estado de São Paulo. Foram utilizados os isolados: IB 01/02, obtido de plântulas de cafeeiro de um viveiro de mudas em Itapira, SP; IB02/02, de mudas de cafeeiro produzidas no Instituto Biológico em Campinas, SP; IB03/02, de plântulas de um viveiro de mudas de Garça, SP; IB04/02, de plântulas do viveiro do IAC, em Campinas, SP e IB05/02, de lavoura com cinco anos de idade no Município de Presidente Prudente, SP. Estes foram cultivados por cinco dias em meio de cultura V-8 em temperatura ambiente, sob luz fluorescente contínua. As suspensões utilizadas nas inoculações foram preparadas por meio da colocação de um filme de água sobre as placas e pela raspagem da superfície do meio contendo os conídios e micélio do fungo, com auxílio de um pincel. Após a filtragem em peneira, as suspensões foram diluídas até conterem aproximadamente 3,5 a $4 \times 10^{4}$ conídios de C. coffeicola $/ \mathrm{mL}$, sendo então aspergidas sobre as faces superior e inferior das folhas.

Após a inoculação, as mudas foram colocadas em casa de vegetação com controle de temperatura ajustado para $25^{\circ} \mathrm{C} \pm 3^{\circ} \mathrm{C}$, na qual permaneceram sob câmara úmida por 72 horas. Após a retirada da câmara úmida, as mudas foram mantidas em casa de vegetação por mais dois dias, sob regime de microaspersão e umidade relativa do ar em torno de $80 \%$, sendo então transferidas para viveiro.

No terceiro ensaio, visando verificar o efeito residual dos fungicidas, as mudas foram inoculadas, novamente, 31 dias após a primeira inoculação, seguindo os procedimentos descritos anteriormente.

Os ensaios foram realizados em delineamento inteiramente casualizado, com 16 repetições, sendo cada repetição representada por uma muda. Avaliou-se a incidência e a severidade da doença nos três experimentos. A incidência foi obtida pela porcentagem de folhas com lesões (\% FL) e a severidade pelo número médio de lesões por folha, no primeiro experimento. Nos demais ensaios, a severidade foi estimada por uma escala de notas (1-6) feita pelos avaliadores no momento da avaliação, calculando-se a nota média da doença, segundo a fórmula "Nota média $=n_{1} \times 1+n_{2} \times 2+n_{3} \times 3+n_{4}$ $\left.\mathrm{x} 4,+\mathrm{n}_{5} \times 5+\mathrm{n}_{6} \times 6\right) / \mathrm{N}^{\prime \prime}$, em que $\mathrm{n}_{1}, \mathrm{n}_{2}, \mathrm{n}_{3,} \mathrm{n}_{4} \mathrm{n}_{5,} \mathrm{n}_{6}$ = número de folhas da planta com as notas 1, 2, 3, 4, 5 e 6; e $\mathrm{N}=$ total de folhas da amostra (MoraIs, 2008). Também foi estimado o Índice de Doença, tomando-se várias folhas representativas de cada nível da escala, que foram fotografadas e desenhadas em filme transparente. A porcentagem de área afetada para cada nível da escala foi estimada por meio da leitura das folhas em aparelho LICor modelo LI-3050A/4, sendo 0 para o nível 1,15\% para o nível 2, 18\% para o nível 3,30\% para o nível 4, 50\% para o nível 5 e $60 \%$ para o nível 6 . O índice de doença foi calculado de acordo com a fórmula "Índice de doença $(\%)=\left(n_{1} \times 0+n_{2} \times 15+n_{3} \times 18+n_{4} \times 30+\right.$ $\left.\mathrm{n}_{5} \times 50+\mathrm{n}_{6} \times 60\right) / \mathrm{N}^{\prime \prime}$, em que $\mathrm{n}_{1}, \mathrm{n}_{2}, \mathrm{n}_{3}, \mathrm{n}_{4,} \mathrm{n}_{5} \mathrm{n}_{6}=$ número de folhas da planta com as notas $1,2,3$, 4, 5 e 6; e $\mathrm{N}=$ total de folhas da amostra (Morais, 2008). No experimento 3 foi calculada a área abaixo da curva de progresso da doença (AACPD) para a variável severidade.

Os dados foram submetidos à análise de variância, após transformações quando necessárias, e as médias comparadas pelo teste de Tukey a $5 \%$ de probabilidade. 


\section{RESULTADOS}

No primeiro experimento, todos os tratamentos reduziram a incidência e a severidade da cercosporiose, aos 23 dias após a inoculação. O oxicloreto de cobre, entretanto, foi menos eficiente que os demais tratamentos. Aos 36 e 46 dias após a inoculação, o tratamento com o oxicloreto de cobre $(69,2$ a $70,6 \%$ de incidência) não diferiu da testemunha sem tratamento (70,4 e 77,9\% deincidência). Aos 46 dias após a inoculação, os tratamentos com epoxiconazol + piraclostrobina e ciproconazol foram os mais eficientes, não apresentando lesões de cercosporiose. Estes tratamentos não diferiram deepoxiconazol, azoxistrobina e carbendazim $(7,7 ; 1,7$ e 13,3\% de icidência), mas diferiram do tratamento com tetraconazol $(15,6 \%)$, que foi menos eficiente. Com exceção do oxicloreto de cobre, todos os demais tratamentos reduziram a severidade da cercosporiose e não diferiram entre si com relação a esta variável, nas três avaliações realizadas (Tabela 1 ).

No segundo ensaio, aos 30 dias após a inoculação, todos os tratamentos promoveram a redução da cercosporiose, porém, os tratamentos com ciproconazol + tiametoxam $(2,9 \%)$ e com azoxistrobina $(1,1 \%)$ foram mais eficientes que os demais. Aos 45 dias após a inoculação, todos os tratamentos reduziram a incidência e a severidade da mancha de olho pardo $(70,7 \%$ de incidência na testemunha), porém, o tratamento com oxicloreto de cobre $(22,9 \%)$ foi menos eficiente que os demais (Tabela 2).

Tabela 1 - Incidência e severidade da cercosporiose do cafeeiro, causada por Cercospora coffeicola, em mudas de café tratadas com diferentes fungicidas. Primeiro experimento.

\begin{tabular}{|c|c|c|c|c|c|c|}
\hline \multirow[t]{2}{*}{ Tratamentos } & \multicolumn{3}{|c|}{$\begin{array}{c}\text { Incidência } \\
\text { (\% folhas com lesões/planta) }\end{array}$} & \multicolumn{3}{|c|}{$\begin{array}{c}\text { Severidade } \\
\text { (número médio de lesões/folha) }\end{array}$} \\
\hline & 23 d.a.i. ${ }^{1}$ & 36 d.a.i. & 46 d.a.i. & 23 d.a.i. $^{1}$ & 36 d.a.i. & 46 d.a.i. \\
\hline Testemunha & $61,5 a^{2,3}$ & $70,4 \mathrm{a}^{2,3}$ & $77,9 \mathrm{a}^{2,3}$ & $3,76 \mathrm{a}^{2,4}$ & $4,38 \mathrm{a}^{2,4}$ & $3,21 \mathrm{a}^{2,4}$ \\
\hline Epoxiconazol & $0,9 \mathrm{c}$ & $5,3 \mathrm{~b}$ & $7,7 \mathrm{bc}$ & $0,01 \mathrm{c}$ & $0,06 \mathrm{~b}$ & $0,09 \mathrm{~b}$ \\
\hline Epoxiconazol + piraclostrobina & $1,9 \mathrm{c}$ & $1,7 \mathrm{~b}$ & $0,0 \mathrm{c}$ & $0,03 \mathrm{c}$ & $0,02 \mathrm{~b}$ & $0,00 \mathrm{~b}$ \\
\hline Tetraconazol & $3,2 c$ & $10,6 \mathrm{~b}$ & $15,6 \mathrm{~b}$ & $0,03 \mathrm{c}$ & $0,19 \mathrm{~b}$ & $0,33 \mathrm{~b}$ \\
\hline Ciproconazol & $4,4 \mathrm{c}$ & $2,6 \mathrm{~b}$ & $0,0 \mathrm{c}$ & $0,04 \mathrm{c}$ & $0,06 \mathrm{~b}$ & $0,00 \mathrm{~b}$ \\
\hline Oxicloreto de cobre & $46,1 \mathrm{~b}$ & $69,2 \mathrm{a}$ & $70,6 \mathrm{a}$ & $2,12 b$ & $3,66 \mathrm{a}$ & $3,12 \mathrm{a}$ \\
\hline Azoxistrobina $^{5}$ & $1,0 \mathrm{c}$ & $1,0 \mathrm{~b}$ & $1,7 \mathrm{bc}$ & $0,01 \mathrm{c}$ & $0,01 \mathrm{~b}$ & $0,02 \mathrm{~b}$ \\
\hline Carbendazim & $1,7 \mathrm{c}$ & $7,3 \mathrm{~b}$ & $13,3 \mathrm{bc}$ & $0,02 \mathrm{c}$ & 0,09 & $0,21 \mathrm{~b}$ \\
\hline $\mathrm{CV}(\%)$ & 42,3 & 43,3 & 40,04 & 42,0 & 29,4 & 26,5 \\
\hline \multicolumn{7}{|c|}{${ }^{1}$ D.a.i. - dias após a inoculação } \\
\hline \multicolumn{7}{|c|}{${ }^{2}$ Análise dos dados transformados para raiz $x+1$} \\
\hline \multicolumn{7}{|c|}{${ }^{3}$ Médias comparadas pelo teste de Tukey a 5\% de probabilidade. } \\
\hline \multicolumn{7}{|c|}{${ }^{4}$ Análise dos dados transformados para raiz $x+0,5$} \\
\hline${ }^{5}$ Produto acrescido com adjuvan & Nimbus a & & & & & \\
\hline
\end{tabular}

Tabela 2 - Incidência, severidade (1-6) e índice de área foliar afetada, da cercosporiose do cafeeiro, causada por Cercospora coffeicola em mudas de cafeeiro tratadas com diferentes fungicidas, aplicados via solo ou na parte aérea. Segundo experimento.

\begin{tabular}{|c|c|c|c|c|}
\hline Tratamentos & $\begin{array}{c}\text { Incidência } \\
\text { (\% folhas com lesões/ } \\
\text { planta) }\end{array}$ & $\begin{array}{c}\text { Incidência } \\
\text { (\% folhas com lesões/ } \\
\text { planta) }\end{array}$ & $\begin{array}{l}\text { Severidade } \\
\text { (notas 1-6) }\end{array}$ & $\begin{array}{l}\text { Índice de área } \\
\text { foliar afetada (\%) }\end{array}$ \\
\hline & 30 d.a.i. ${ }^{1}$ & \multicolumn{3}{|c|}{45 d.a.i. } \\
\hline Testemunha & $27,9 \mathrm{a}^{2,3}$ & $70,7 \mathrm{a}^{2,3}$ & $2,43 \mathrm{a}^{2,4}$ & $16,5 \mathrm{a}^{2}$ \\
\hline Ciproconazol + tiametoxam & $2,9 \mathrm{c}$ & $3,2 \mathrm{c}$ & $1,03 \mathrm{~b}$ & $0,49 \mathrm{c}$ \\
\hline Triadimenol & $7,1 \mathrm{~b}$ & $6,9 c$ & $1,09 \mathrm{~b}$ & $1,14 \mathrm{c}$ \\
\hline Azoxistrobina & $1,1 \mathrm{c}$ & $0,0 \mathrm{c}$ & $1,00 \mathrm{~b}$ & $0,00 \mathrm{c}$ \\
\hline Oxicloreto de cobre & $10,2 \mathrm{~b}$ & $22,9 \mathrm{~b}$ & $1,27 \mathrm{~b}$ & $3,64 \mathrm{~b}$ \\
\hline $\mathrm{CV}(\%)$ & 55,9 & 59,6 & 23,7 & 28,14 \\
\hline
\end{tabular}

${ }^{1}$ D.a.i. - dias após a inoculação

${ }^{2}$ Análise dos dados transformados para raiz $x+1$

${ }^{3}$ Médias comparadas pelo teste de Tukey a $5 \%$ de probabilidade.

${ }^{4}$ Análise dos dados transformados para raiz $\mathrm{x}+0,5$ 
Tabela 3 - Incidência, avaliada pela porcentagem de folhas com lesões por planta, e severidade, avaliada por uma escala de notas (1-6), da cercosporiose do cafeeiro, causada por Cercospora coffeicola em mudas tratadas com diferentes fungicidas, aplicados via solo ou na parte aérea. Terceiro experimento.

\begin{tabular}{|c|c|c|c|c|c|c|c|c|}
\hline \multirow{3}{*}{$\begin{array}{l}\text { Tratamentos (doses dos produ- } \\
\text { tos comerciais/100 L de água) }\end{array}$} & \multicolumn{4}{|c|}{ Incidência } & \multicolumn{4}{|c|}{ Severidade (Notas 1-6) } \\
\hline & \multicolumn{4}{|c|}{ (\% folhas com lesões/planta) } & & & & \\
\hline & 20 d.a.i. $^{1}$ & 32 d.a.i & 49 d.a.i. & 56 d.a.i. & 20 d.a.i. & 32 d.a.i & 49 d.a.i. & 56 d.a.i. \\
\hline 1. Testemunha & $19,8 \mathrm{a}^{2,3}$ & $40,8 \mathrm{a}^{2,3}$ & $57,8 \mathrm{a}^{2,3}$ & $65,2 \mathrm{a}^{2,3}$ & $1,37 \mathrm{a}^{2}$ & $1,85 \mathrm{a}^{2}$ & $2,22 \mathrm{a}^{2}$ & $2,39 \mathrm{a}^{2}$ \\
\hline 2. Triadimenol + dissulfoton & $0,0 \mathrm{c}$ & $3,5 \mathrm{~cd}$ & 18,8 cdef & 39,4 abcde & $1,00 \mathrm{~b}$ & $1,07 \mathrm{bc}$ & $1,37 \mathrm{bc}$ & $1,67 \mathrm{bc}$ \\
\hline 3. Ciproconazol + tiametoxam & $0,5 \mathrm{c}$ & $8,2 \mathrm{bcd}$ & $7,8 \mathrm{f}$ & $18,0 \mathrm{e}$ & $1,00 \mathrm{~b}$ & $1,11 \mathrm{bc}$ & $1,18 \mathrm{bc}$ & $1,30 \mathrm{c}$ \\
\hline 4. Triadimenol & $6,9 c$ & $16,4 \mathrm{~b}$ & 24,9 bcde & 33,0 bcde & $1,00 \mathrm{~b}$ & $1,34 \mathrm{~b}$ & $1,65 \mathrm{~b}$ & $1,64 \mathrm{bc}$ \\
\hline 5. Epoxiconazol & $0,0 \mathrm{c}$ & $2,3 \mathrm{~d}$ & 18,9 cdef & 26,5 cde & $1,00 \mathrm{~b}$ & $1,03 \mathrm{c}$ & $1,36 \mathrm{bc}$ & $1,51 \mathrm{bc}$ \\
\hline 6. Epoxiconazol + piraclostrobina & $0,0 \mathrm{c}$ & $1,8 \mathrm{~d}$ & 10,6 def & $19,8 \mathrm{e}$ & $1,00 \mathrm{~b}$ & $1,02 \mathrm{c}$ & $1,14 \mathrm{c}$ & $1,30 \mathrm{c}$ \\
\hline 7. Tetraconazol & $0,6 \mathrm{c}$ & $9,9 \mathrm{bcd}$ & $27,9 \mathrm{bcd}$ & 40,8 abcd & $1,00 \mathrm{~b}$ & $1,15 \mathrm{bc}$ & $1,60 \mathrm{bc}$ & $1,90 \mathrm{ab}$ \\
\hline 8. Ciproconazol & $2,4 \mathrm{c}$ & $11,5 \mathrm{bcd}$ & 17,9 cdef & 23,2 cde & $1,03 \mathrm{~b}$ & $1,19 \mathrm{bc}$ & $1,43 \mathrm{bc}$ & $1,49 \mathrm{bc}$ \\
\hline 9. Oxicloreto de cobre & $10,4 \mathrm{~b}$ & 38,9 a & $46,5 \mathrm{ab}$ & $51,9 \mathrm{ab}$ & $1,11 \mathrm{~b}$ & $1,72 \mathrm{a}$ & $2,34 \mathrm{a}$ & $2,45 \mathrm{a}$ \\
\hline 10. Azoxistrobina & $1,1 \mathrm{c}$ & $14,9 \mathrm{bc}$ & $27,9 \mathrm{bc}$ & 39,3 abcde & $1,01 \mathrm{~b}$ & $1,20 \mathrm{bc}$ & $1,47 \mathrm{bc}$ & $1,81 \mathrm{bc}$ \\
\hline 11. Carbendazim & $0,0 \mathrm{c}$ & $5,8 \mathrm{~cd}$ & 10,4 ef & 21,3 de & $1,00 \mathrm{~b}$ & $1,10 \mathrm{bc}$ & $1,19 \mathrm{bc}$ & $1,33 \mathrm{c}$ \\
\hline 12. Testemunha 2 & - & - & 20,5 cdef & $44,7 \mathrm{abc}$ & & & $1,23 \mathrm{bc}$ & $1,81 \mathrm{~b}$ \\
\hline $\mathrm{CV}(\%)$ & 65,03 & 49,00 & 38,26 & 30,1 & 10,0 & 19,5 & 27,5 & 28,18 \\
\hline
\end{tabular}

${ }^{1}$ D.a.i. - dias após a inoculação

${ }^{2}$ Análise dos dados transformados para raiz $\mathrm{x}+1$

${ }^{3}$ Médias comparadas pelo teste de Tukey a 5\% de probabilidade.

Tabela 4 - Área foliar afetada e área abaixo da curva de progresso da incidência, severidade e da área foliar afetada da cercosporiose do cafeeiro, causada por Cercospora coffeicola em mudas tratadas com diferentes fungicidas, aplicados via solo ou na parte aérea. Julho a agosto de 2003.

\begin{tabular}{|c|c|c|c|c|c|}
\hline \multirow{2}{*}{$\begin{array}{l}\text { Tratamentos (doses dos produtos } \\
\text { comerciais/100 L de água) }\end{array}$} & \multicolumn{3}{|c|}{ Área foliar afetada $(0-60 \%)$} & \multicolumn{2}{|c|}{ Área abaixo da curva de progresso } \\
\hline & 20 d.a.i. ${ }^{1}$ & 32 d.a.i & 49 d.a.i. & 56 d.a.i. & Severidade \\
\hline 1. Testemunha & $4,48 \mathrm{a}^{2,3}$ & $9,93 \mathrm{a}^{2,3}$ & $14,20 \mathrm{a}^{2,3}$ & $15,72 a b^{2,3}$ & $63,9 \mathrm{a}^{2,3}$ \\
\hline 2. Triadimenol + dissulfoton & $0,00 \mathrm{~d}$ & $0,84 \mathrm{c}$ & 4,02 bcd & $7,82 \mathrm{~cd}$ & $42,3 \mathrm{bc}$ \\
\hline 3. Ciproconazol + tiametoxam & $0,08 \mathrm{~d}$ & $1,31 \mathrm{bc}$ & $1,91 \mathrm{~d}$ & $3,70 \mathrm{~d}$ & $37,7 \mathrm{c}$ \\
\hline 4. Triadimenol & $1,36 \mathrm{bc}$ & $3,91 \mathrm{~b}$ & $7,52 \mathrm{~b}$ & $7,47 \mathrm{~cd}$ & $49,1 \mathrm{~b}$ \\
\hline 5. Epoxiconazol & $0,00 \mathrm{~d}$ & $0,35 \mathrm{c}$ & $4,07 \mathrm{bcd}$ & $5,84 \mathrm{~cd}$ & $42,0 \mathrm{bc}$ \\
\hline 6. Epoxiconazol + piraclostrobina & $0,00 \mathrm{~d}$ & $0,27 c$ & $1,70 \mathrm{~d}$ & $3,68 \mathrm{~d}$ & $37,2 \mathrm{c}$ \\
\hline 7. Tetraconazol & $0,08 \mathrm{~d}$ & $1,75 \mathrm{bc}$ & $6,63 \mathrm{bc}$ & $10,36 \mathrm{abc}$ & $47,0 \mathrm{bc}$ \\
\hline 8. Ciproconazol & $0,38 \mathrm{bcd}$ & $2,17 \mathrm{bc}$ & $5,14 \mathrm{bcd}$ & $5,64 \mathrm{~cd}$ & $43,5 \mathrm{bc}$ \\
\hline 9. Oxicloreto de cobre & $1,60 \mathrm{~b}$ & $7,91 \mathrm{a}$ & $15,65 \mathrm{a}$ & $16,84 \mathrm{a}$ & $64,0 \mathrm{a}$ \\
\hline 10. Azoxistrobina & $0,19 \mathrm{c}$ & $2,43 \mathrm{bc}$ & $5,59 \mathrm{bcd}$ & $9,07 \mathrm{bcd}$ & $44,3 \mathrm{~b}$ \\
\hline 11. Carbendazim & $0,00 \mathrm{~d}$ & $1,03 \mathrm{c}$ & $2,11 \mathrm{~d}$ & $4,22 \mathrm{~d}$ & $38,5 \mathrm{c}$ \\
\hline 12. Testemunha 2 & & & $3,56 \mathrm{bcd}$ & $9,89 \mathrm{abc}$ & \\
\hline $\mathrm{CV}(\%)$ & 28,25 & 33,72 & 34,55 & 30,07 & 20,2 \\
\hline
\end{tabular}

${ }^{1}$ D.a.i. - dias após a inoculação

${ }^{2}$ Análise dos dados transformados para raiz $\mathrm{x}+1$

${ }^{3}$ Médias comparadas pelo teste de Tukey a $5 \%$ de probabilidade

No terceiro ensaio, aos 20 dias após a inoculação, todos os tratamentos reduziram a incidência da cercosporiose, entretanto, assim como nos experimentos anteriores, o oxicloreto de cobre foi menos eficiente que os demais. Na segunda avaliação, aos 32 dias após a inoculação, os tratamentos com epoxiconazol e epoxiconazol + piraclostrobina apresentaram as menores incidências da doença $(2,3$ e 1,8\%, respectivamente), mas não diferiram dos tratamentos com triadimenol + dissulfoton $(3,5 \%)$, ciproconazol + tiametoxam $(8,2 \%)$, tetraconazol $(9,9 \%)$ e ciproconazol $(11,5 \%)$. Nesta avaliação, o tratamento com o oxicloreto decobre (38,9\%) não diferiu da testemunha (40,8\%). Aos 49 dias após a primeira inoculação e aos 17 dias após a segunda inoculação, o tratamento com a menor incidência da cercosporiose foi com ciproconazol + tiametoxam via solo $(7,8 \%)$. Este tratamento não diferiu dos tratamentos com triadimenol + 
disulfoton (18,9\%), epoxiconazol (18,9\%), epoxiconazol + piraclostrobina (10,8\%), ciproconazol $(17,9 \%)$ e carbendazim (10,4\%). Na última avaliação, aos 56 dias após a primeira inoculação, os tratamentos com as menores incidências de cercosporiose foram com ciproconazol + tiametoxam via solo (18\%) e epoxiconazol + piraclostrobina (19,8\%). Esses tratamentos não diferiram dos tratamentos com epoxiconazol (26,5\%), ciproconazol (23,2\%), carbendazim (21,3\%), triadimenol + dissulfoton $(39,4 \%)$, triadimenol via solo (33,0\%) eaxozistrobina (39,3\%). Nesta avaliação os dois últimos tratamentos também não diferiram das testemunhas, tanto a que sofreu duas inoculações, como da que recebeu apenas a segunda inoculação.

Com relação à severidade, na primeira avaliação, todos os tratamentos diferiram da testemunha, mas não diferiram entre si. Aos 32 dias após a inoculação, os tratamentos com epoxiconazol $(1,03)$ e epoxiconazol + piraclostrobina $(1,02)$ tiveram os menores índices de severidade da cercosporiose, mas não diferiram dos tratamentos com triadimenol + dissulfoton $(1,07)$, ciproconazol + tiametoxan $(1,11)$, tetraconazol $(1,15)$, ciproconazol $(1,19)$, azoxistrobina $(1,72)$ e carbendazim $(1,10)$ (Tabela 3$)$. Aos 49 dias após a primeira inoculação, o tratamento com epoxiconazol + piraclostrobina $(1,14)$ teve o menor índice de severidade da doença. Este tratamento diferiu, além da testemunha $(2,22)$, apenas dos tratamentos com triadimenol $(1,65)$ e oxicloreto de cobre $(1,43)$. Este último não diferiu da testemunha. $\mathrm{Na}$ quarta avaliação, os tratamentos com ciproconazol + tiametoxam $(1,51)$, epoxiconazol + piraclostrobina $(1,33)$ e carbendazim $(1,33)$ tiveram os menores índices de severidade da doença. Estes tratamentos diferiram dos tratamentos com tetraconazol $(1,90)$ e oxicloreto de cobre $(2,45)$ menos eficientes, e foram também significativamente diferentes da testemunha que recebeu duas inoculações $(2,39)$ e da que recebeu apenas a segunda inoculação $(1,81)$ (Tabela 3$)$.

Na primeira avaliação, 20 dias após a inoculação, todos os tratamentos reduziram a área foliar afetada, porém os tratamentos com triadimenol e oxicloreto de cobre foram menos eficientes que os demais. $\mathrm{Na}$ avaliação realizada 32 dias após a inoculação, com exceção do oxicloreto de cobre, os demais tratamentos reduziram a área foliar afetada. Nessa avaliação, os tratamentos com epoxiconazol + piraclostrobina $(0,27 \%)$, epoxiconazol $(0,35 \%)$, triadimenol + dissulfoton $(0,84 \%)$ e carbendazim $(1,03 \%)$ tiveram os menores índices de área foliar afetada. Na terceira e quarta avaliações, realizadas aos 49 e 56 dias após a inoculação, os tratamentos com ciproconazol + tiametoxam $(1,91$ e $3,70 \%)$, epoxiconazol + piraclostrobina $(1,70 \mathrm{e} 3,68 \%)$ ecarbendazim $(2,11$ e $4,22 \%)$ resultaram nas menores áreas foliares afetadas (testemunha com 14,20 e 15,72\%). Emambas as avaliações, o tratamento com o oxicloreto de cobre não diferiu da testemunha.
Aos 49 dias após a primeira inoculação, com exceção do oxicloreto de cobre, todos os tratamentos diferiram da testemunha que recebeu duas inoculações $(14,20 \%)$, mas não diferiram da testemunha que recebeu só uma inoculação $(3,68 \%)$. Na avaliação aos 56 dias após a inoculação, os tratamentos com tetraconazol e oxicloreto de cobre não diferiram da testemunha com duas inoculações $(15,72 \%)$. Os tratamentos com ciproconazol + tiametoxam $(3,70 \%)$ e epoxiconazol + piraclostrobina $(3,68 \%)$ e carbendazim $(4,27 \%)$ diferiram de ambas as testemunhas, indicando que têm um poder residual relativamente elevado (Tabela 4).

Os tratamentos com epoxiconazol + piraclostrobina $(37,2)$, ciproconazol + tiametoxam $(37,7)$ e com carbendazim $(38,5)$ foram os que tiveram os menores índices de área abaixo da curva de progresso (AACP) da severidade da cercosporiose, que mediu o resultado do controle imediato da doença e também o efeito residual dos fungicidas, pois foram realizadas duas inoculações sem a reaplicação dos produtos. Estes tratamentos não diferiram dos com triadimenol + dissulfoton (42,3), epoxiconazol $(42,0)$ e ciproconazol $(43,5)$ (Tabela 4.).

\section{DISCUSSÃO}

Neste estudo, o oxicloreto de cobre foi menos eficiente e teve menor efeito residual que os demais produtos testados. Os fungicidas cúpricos estão entre os primeiros produtos químicos utilizados para o controle da ferrugem do cafeeiro, tendo também ação contra a cercosporiose (MATiEllo; AlmeidA, 2006). A menor eficiência do oxicloreto de cobre observada no presente estudo pode ser devida às irrigações frequentes e intensas que incidiram sobre as mudas, durante a condução dos experimentos. TöFoli et al. (2002) observaram que mudas de café tratadas com o oxicloreto de cobre e que receberam simulação de chuva por 60 e 120 minutos tiveram o controle da ferrugem reduzido. Também a forma de inoculação do patógeno pode ter interferido nos resultados. Ofungo foi inoculado nas faces superiore inferior das folhas, enquanto os cúpricos geralmente se depositam apenas na face superior das folhas, não penetrando nelas (Matiello; Almeida, 2006). PozzA et al. (1997) observaram menor eficiência desse fungicida que a do tiofanato metílico, do benomil e do tebuconazol e, no estudo de PATRICIO et al. (2008) o produto teve efeito inferior ao da azoxistrobina, como no presente trabalho. Em condições de campo, Matiello; Almeida (2006) relataram efeito parcial do oxicloreto de cobre no controle da cercosporiose e da ferrugem do cafeeiro.

Os produtos formulados com triadimenol, ou a mistura de tradimenol + dissulfotom, aplicados via 
solo neste estudo foram moderadamente eficientes para o controle da cercosporiose, embora tenham apresentado menor efeito residual que alguns dos demais triazóis testados. O triadimenol está entre os primeiros triazóis desenvolvidos para o controle da ferrugem do cafeeiro na década de 1980 (Matiello; Almeida, 2006), mas há poucas informações sobre o seu efeito sobre a cercosporiose do cafeeiro. Assim como no presente estudo, em outro patossistema semelhante, da mancha preta do abacate, causada por Pseudocercospora purpurea, foi obtido o controle da doença com triadimenol aplicado via solo, complementado com uma aplicação foliar de oxicloreto de cobre ou pela injeção de triadimenol no troco das plantas (DuvenHage, 1994). Naquele trabalho, o tratamento com oxicloreto de cobre seguido pela aplicação no solo do triadimenol foi pouco eficiente, provavelmente porque o produto não foi translocado a tempo para as partes vegetativas da planta expostas ao patógeno. Em condições de campo, o triadimenolé aplicado no solo no mês de novembro, mas, como no presente estudo o produto teve efeito residual moderado para a cercosporiose, supõe-se que, na época em que ocorrem as epidemias mais graves de cercosporiose, janeiro a fevereiro/março, a sua concentração já não seja suficiente para proporcionar o controle do patógeno em níveis adequados. Por esta razão, frequentemente são necessárias aplicações foliares complementares de outros fungicidas para o controle da mancha de olho pardo, embora tenha sido demonstrado neste estudo que o produto tem efeito sobre C. coffeicola.

Os produtos formulados com o ciproconazol, aplicado isoladamente na parte aérea, ou no solo, combinado ao tiametoxam, foram eficientes e, especialmente o último, tiveram um bom efeito residual para o controle da cercosporiose nas mudas de cafeeiro. Assim como neste trabalho, em estudo anterior de Culbreath et al. (1995), o ciproconazol foi eficiente para o controle de Cercosporidium personatum, agente causal da cercosporiose do amendoim. Também o ciproconazol é utilizado para o controle de Cercospora beticola em culturas de beterraba na Grécia (KARADIMOS et al., 2005). O ciproconazol está listado entre os triazóis tradicionalmente empregados para o controle da ferrugem do cafeeiro em condições de campo (Matiello; Almeida, 2006), mas apenas a mistura de ciproconazol com axozistrobina é recomendada para o controle simultâneo da ferrugem e da cercosporiose (Matiello; Almeida, 2006), provavelmente porque, em condições de campo, seja relativamente difícil medir o efeito desse triazol sobre a cercosporiose. No campo, as epidemias de ferrugem e outros distúrbios podem ser muito severos, causando a queda das folhas antes de elas serem atingidas pela cercosporiose.
No presente estudo, os fungicidas epoxiconazol e epoxiconazol + piraclostrobina foram eficientes e tiveram um efeito residual relativamente longo sobre o controle da cercosporiose. Assim como neste trabalho, RACCA; JÖRG (2007) verificaram que o epoxiconazol, em apenas uma aplicação, esteve entre os fungicidas que mais reduziram a incidência e atrasaram a epidemia da cercosporiose, causada por C. beticola, em beterraba açucareira. A mistura epoxiconazol + piraclostrobina é recomendada para o controle simultâneo da ferrugem e da cercosporiose (Matiello; Almeida, 2006).

Otetraconazol proporcionou o controle da cercosporiose nas mudas de cafeeiro, porém teve um efeito residual um pouco menor que o dos demais triazóis. O resultado é semelhante ao verificado por KHAN; SмIтH (2005), que observaram que o tetraconazol esteve entre os produtos eficientes para o controle de C. beticola em beterraba açucareira, sendo o triazol utilizado na Grécia para este mesmo patossistema (KARADIMOs et al., 2005).

Neste estudo, a azoxistrobina foi colocada como padrão para o controle da cercosporiose, fungicida que tem ação efetiva para o controle da cercosporiose na cultura da beterraba (ANESIADIs et al., 2003; KARADIMOs et al., 2005), e foi eficiente para o controle de C. coffeicola em cafeeiros (PAtricio et al., 2008). O produto não teve um efeito residual muito grande, o que era esperado, pois as estrobilurinas são utilizadas preventivamente para o controle de doenças (BARTLETT et al., 2002) e também porque o tempo em que foi medido o efeito residual pode ser considerado longo, até 56 dias após a aplicação, tempo no qual o produto pode ter sido degradado, especialmente porque as mudas foram submetidas a irrigações frequentes no viveiro.

Neste estudo, o carbendazim também foi incluído como padrão para o controle da cercosporiose, tendo demonstrado um bom controle e um efeito residual relativamente elevado. O resultado era esperado, pois Pozza et al. (1997) já havia verificado o controle deste patógeno com o tiofanato metílico e o benomil, fungicidas do grupo dos benzimindazóis. Estes fungicidas, entretanto, apresentam alto risco de desenvolvimento de resistência (GHINI; Kimati, 2000), e com histórico de perda de eficiência em patossistemas semelhantes, como C. beticola em beterraba (KHAN; SMITH, 2005). Portanto, sugere-se que fungicidas deste grupo sejam aplicados em esquema de rotação ou misturados a fungicidas protetores para evitar o desenvolvimento de populações de $C$. coffeicola resistentes.

Embora os triazóis tenham sido eficientes no controle da cercosporiose neste estudo, foi possível observar nos tratamentos com ciproconazol e epoxiconazol sintomas de fitotoxicidade, com ligeiro encurtamento de internódios e encurvamento das 
folhas, que foram reversíveis nas brotações posteriores, sugerindo-se que os triazóis não sejam aplicados em mudas. Na literatura há relatos de que em lavouras tratadas via solo por vários anos consecutivos com fungicidas do grupo dos triazóis misturados a inseticidas, visando o controle da ferrugem e do bicho mineiro, poderiam ser mais afetadas pela cercosporiose (ZАMBOLIM et al., 1997; ZАMBOLIM et al., 2005). Neste estudo, observou-se que os fungicidas triazóis e suas misturas com inseticidas, tanto os aplicados via solo como na parte aérea, reduziram a incidência e a severidade da cercosporiose nas mudas de cafeeiro. Supõe-se, portanto, que o fenômeno observado no campo possa ser devido à presença de quantidade insuficiente do princípio ativo na planta na época em que a cercosporiose causa os maiores prejuízos, especialmente considerando que os fungicidas e inseticidas são aplicados no solo em novembro ou dezembro e a cercosporiose é mais grave nos meses de janeiro a março. Também ocorre um intenso crescimento vegetativo nesta época do ano e pode não haver produto suficiente nas folhas mais jovens que proporcione o controle da doença em níveis satisfatórios. Os resultados deste trabalho, portanto, não excluem a necessidade de acompanhamento da epidemia da cercosporiose no campo e, se necessário, de controle complementar da doença de janeiro a março, época em que pode causar os maiores prejuízos.

\section{CONCLUSÕES}

Os produtos aplicados via solo: ciproconazol + tiametoxam, triadimenol + dissulfotom e triadimenol, podem reduzir a incidência e a severidade da cercosporiose em mudas de cafeeiro.

Os triazóis aplicados na parte aérea: epoxiconazol, ciproconazol, tetraconazol, a mistura epoxiconazol + piraclostrobina e o carbendazim são eficientes para o controle da cercosporiose do cafeeiro.

Os triazóis, com excessão do tetraconazole, podem ser fitotóxicos a mudas de cafeeiro, no entanto, os efeitos são reversíveis.

O ciproconazol + tiametoxam, aplicado via solo, o epoxiconazol + piraclostrobina e o carbendazim, aplicados via foliar, têm longo efeito residual sobre o controle da cercosporiose em cafeeiro.

\section{REFERÊNCIAS}

ANESIADIS, T.; KARAOGLANIDIS, G.S.; TZAVELLAKLONARI, K. Protective, curative and erradicant activity of the strobilurin fungicide azoxystrobin against Cercospora beticola and Erisiphe betae. Journal of Phytopathology, v.151, p.647-651, 2003.
BARTLETT, D.W.; CLOGH, J.M.; HALL, A.A.; HAMER, M.; PARR-DOBRZANSKY, B. The strubilurin fungicides. Pest Management Science, v.58, p.649-662, 2002.

CARVALHO, V.L.; CUNHA R.L.; CHALFOUN S.M. Manejo ecológico das principais doenças do cafeeiro. Informe Agropecuário, v. 23, p.101-114, 2002.

CULBREATH, A.K.; BRENNEMAN, T.B.; BONDARI, K.; REYNOLDS, K.L.; McLEAN, H.S. Late leaf spot, southern stem rot, and peanut yield responses to rates of cyproconazole and chlorothalonil applied alone and in combination. Plant Disease, v.79, p.1121-1125, 1995.

DUVENHAGE, J.A. Control of black spot of avocado by organic and inorganic fungicides. South African Avocado Growers Association Yearbook, v.17, p.49-52, 1994.

GHINI, R.; KIMATI, H. Resistência de fungos a fungicidas. Jaguariúna: Embrapa Meio Ambiente, 2000. 78p.

GODOY, C.V.; BERGAMIN FILHO, A.; SALGADO, C.L. Doenças do cafeeiro. In: KIMATI, H.; AMORIM, L.; REZENDE, J.A.M.; BERGAMIN FILHO, A.; CAMARGO, L.E.A. (Ed.). Manual de fitopatologia. 3. ed. São Paulo: Editora Ceres, 1997. p.184-200. vol. 2: Doenças das plantas cultivadas.

KARADIMOS, D.A.; KARAOGLANIDIS, G.S.; TZAVELLA-KLONARI, K. Biological activity and phsical modes of action of the Qo inhibitor fungicides trifloxstrobin and praclostrobin against Cercospora beticola. Crop Protection, v.24, p.23-29, 2005.

KHAN, M.F.R.; SMITH, L. Evaluating fungicides for controlling Cercospora leaf spot on sugar beet. Crop Protection, v.24, p.79-86, 2005.

MATIELLO, J.B. O café - do cultivo ao consumo. São Paulo: Publicações Globo Rural, 1991. 320p.

MATIELLO, J.B.; ALMEIDA, S.R. A ferrugem do cafeeiro no Brasil e seu controle. Varginha: PROCAFÉ, 2006. 98p.

MORAES, S.A. de Quantificação de doenças de plantas. 2007. Artigo em Hypertexto. Disponível em: <http:// www.infobibos.com/Artigos/2007_1/doencas/index. htm>. Acesso em: 2 mai. 2008.

PATRICIO, F.R.A; ALMEIDA, I.M.G.; BARROS, B.C.; SANTOS, A.S.; FRARE, P.M. Effectiveness of acibenzolar-S-methyl, fungicides and antibiotics for the control of brown eye spot, bacterial blight, brown leaf spot and coffee rust in coffee. Annals of Applied Biology, n.152, p.29-39, 2008.

POZZA, A.A.A.; ZAMBOLIM, L.; POZZA, E.A.; COSTA H.; VALE F.X.R. Controle químico da mancha de olho pardo (Cercospora coffeicola) do cafeeiro em condições de viveiro. Fitopatologia Brasileira, v.22, p.543-545, 1997. 
POZZA, A.A.A.; MARTINEZ, H.E.P.; POZZA, E.A.; CAIXETA S.L.; ZAMBOLIM L. Intensidade da manchade-olho-pardo em mudas de cafeeiro em função de doses de N e K em solução nutritiva. Summa Phytopathologica, v.26, n.1, p.29-33, 2000.

RACCA, P.; JÖRG, E. CERCBET 3 - a forecaster for epidemic development of Cercospora beticola. Bulletin OEPP/EPPO, v.37, p.344-349, 2007.

THOMAZIELLO, R.A., FAZUOLI, L.C., PEZZOPANE, J.R., FAHL, J.I., CARELLI, M.L.C. Café arabica: cultura e técnicas de produção. Campinas: Instituto Agronômico, 2000. 82p. (Boletim Técnico, n.187).

TÓFOLI, J.G.; OLIVEIRA, S.H.F.; DOMINGUES, R.J.; SANTOS J.M.F.; MARTINS, E.M.F. Desempenho de azoxystrobin no controle da ferrugem do cafeeiro sob condições de chuva induzida. Arquivos do Instituto Biológico, São Paulo, v.69, n.3, p.93-96, 2002.

ZAMBOLIM L.; VALE F.X.R.; PEREIRA A.A.; CHAVES G.M. Café. In: VALE F.X.R., ZAMBOLIM L (Ed.). Controle de doenças de plantas. Viçosa: Editora Universidade Federal de Viçosa, 1997. p.83-180.

ZAMBOLIM, L.; VALE, F.X.R.; ZAMBOLIM, E.M. Doenças do cafeeiro. In: KIMATI, H.; AMORIM, L.; REZENDE, J.A.M.; BERGAMIN FILHO, A.; CAMARGO, L.E.A. (Ed.). Manual de fitopatologia. 4.ed. São Paulo: Editora Ceres, 2005. p.165-180. vol. 2: Doenças das plantas cultivadas.

Recebido em 21/7/09

Aceito em 23/2/11 